\title{
Feasibility of CXCR4-Directed Radioligand Therapy in Advanced Diffuse Large B-Cell Lymphoma
}

\author{
Constantin Lapa ${ }^{1}$, Heribert Hänscheid ${ }^{1}$, Malte Kircher ${ }^{1}$, Andreas Schirbel ${ }^{1}$, Gerd Wunderlich ${ }^{2}$, Rudolf A. Werner ${ }^{1}$, \\ Samuel Samnick ${ }^{1}$, Jörg Kotzerke ${ }^{2}$, Hermann Einsele ${ }^{3}$, Andreas K. Buck ${ }^{1}$, Hans-Jürgen Wester ${ }^{4}$, and \\ Götz Ulrich Grigoleit ${ }^{3}$ \\ ${ }^{I}$ Department of Nuclear Medicine, University Hospital Würzburg, Würzburg, Germany; ${ }^{2}$ Department of Nuclear Medicine, University \\ Hospital Dresden, Dresden, Germany; ${ }^{3}$ Division of Hematology and Medical Oncology, Department of Internal Medicine II, \\ Universitätsklinikum Würzburg, Würzburg, Germany; and ${ }^{4}$ Pharmaceutical Radiochemistry, Technische Universität München, \\ Munich, Germany
}

\begin{abstract}
We have recently reported on our experience with $\mathrm{C}-\mathrm{X}-\mathrm{C}$-motif chemokine receptor 4 (CXCR4)-directed radioligand therapy (RLT) in multiple myeloma and acute leukemia. Methods: Six patients with heavily pretreated relapsed diffuse large B-cell lymphoma (3 men, 3 women; aged, $54 \pm 8$ y) underwent CXCR4-directed $\mathrm{RLT}$ in combination with conditioning chemotherapy and allogeneic stem cell transplantation. In 2 patients, radioimmunotherapy targeting CD20 or CD66 was added to enhance antilymphoma activity. Endpoints were incidence and severity of adverse events, progression-free survival, and overall survival. Results: RLT and additional radioimmunotherapy were well tolerated, without any acute adverse events or changes in vital signs. Successful engraftment was recorded after a median of $11 \mathrm{~d}$ (range, 9-13 d). Of the 4 patients who were available for follow-up (one patient died of CNS aspergillosis $29 \mathrm{~d}$ after RLT and another of sepsis in aplasia $34 \mathrm{~d}$ after RLT), CXCR4-directed RLT resulted in a partial response in two (both treated with additional radioimmunotherapy) and a mixed response in the remaining two. The response duration was rather short-lived, with a median progression-free survival of $62 \mathrm{~d}$ (range, 29-110 d) and a median overall survival of $76 \mathrm{~d}$ (range, 29-334 d). Conclusion: CXCR4-directed RLT (in combination with additional radioimmunotherapy) is feasible as a conditioning regimen before allogeneic stem cell transplantation in diffuse large B-cell lymphoma.
\end{abstract}

Key Words: chemokine receptors; CXCR4; lymphoma; DLBCL; radioligand therapy

J Nucl Med 2019; 60:60-64

DOI: 10.2967/jnumed.118.210997

D

iffuse large B-cell lymphoma (DLBCL) is the most common histologic subtype of non-Hodgkin lymphoma, accounting for approximately $25 \%$ of all cases (1). Systemic chemotherapy, immunotherapy with the recombinant anti-CD20 antibody rituximab, and external radiotherapy are the mainstay of therapy and achieve 10 -y survival rates of $40 \%-50 \%$ (2). However, patients with

Received Mar. 6, 2018; revision accepted May 8, 2018.

For correspondence or reprints contact: Constantin Lapa, Department of Nuclear Medicine, Universitätsklinikum Würzburg, Oberdürrbacher Strasse 6, 97080 Würzburg, Germany.

E-mail: lapa_c@ukw.de

Published online May 18, 2018.

COPYRIGHT (C) 2019 by the Society of Nuclear Medicine and Molecular Imaging. refractory or relapsed disease have a worse prognosis, and secondline chemotherapy is administered to achieve a second response before high-dose chemotherapy and subsequent stem cell transplantation (SCT).

C-X-C-motif chemokine receptor 4 (CXCR4) is overexpressed on lymphoma cells (3-6) and has been identified as a potential drug target. Wester et al. successfully developed a radiolabeled CXCR4 ligand $\left({ }^{68} \mathrm{Ga}\right.$-pentixafor) for PET imaging $(5,7,8)$ that visualizes CXCR4 expression in patients with hematologic and solid malignancies (5,9-17). Recently, we and others also reported on experience with ${ }^{177} \mathrm{Lu}$ - and ${ }^{90} \mathrm{Y}$-labeled pentixather (18), the therapeutic counterpart of ${ }^{68} \mathrm{Ga}$-pentixafor for CXCR4-targeted radioligand therapy (RLT) in acute leukemia (19) and in patients with advanced multiple myeloma $(20,21)$. In this article, we evaluate the feasibility of CXCR4-directed RLT as part of the conditioning regimen before allogeneic SCT in patients with relapsed DLBCL of very advanced stage.

\section{MATERIALS AND METHODS}

\section{Subjects}

Six patients ( 3 men, 3 women; aged, $54 \pm 8$ y) with relapsed, refractory DLBCL were included in the study. All patients had undergone multiple lines of previous treatment (2-6 lines of treatment; median, 3 lines) and presented with refractory, progressive disease.

Given the lack of alternative treatments in this advanced disease stage, experimental CXCR4-directed treatment was offered to the patients on a compassionate-use basis (German Drug Act, $\S 13,2$ b) in compliance with $\S 37$ of the Declaration of Helsinki as part of the conditioning regimen before repeated allogeneic SCT. Treatment was approved by the clinical ethics committee of our institution. All subjects gave written informed consent before therapy. Patient characteristics are depicted in Table 1.

\section{${ }^{68}$ Ga-Pentixafor Imaging}

CXCR4 expression was confirmed in all patients using ${ }^{68} \mathrm{Ga}$-pentixafor PET/CT on a dedicated scanner (Biograph mCT 64; Siemens Medical Solutions). CXCR4-directed PET/CT imaging was performed $60 \mathrm{~min}$ after injection of $62-165 \mathrm{MBq}$ (median, $95 \mathrm{MBq}$ ) of ${ }^{68} \mathrm{Ga}-$ pentixafor as previously described $(21) .{ }^{68} \mathrm{Ga}$-pentixafor-positive lesions were visually determined as focally increased tracer retention as compared with surrounding normal tissue or contralateral structures.

\section{Pretherapeutic Dosimetry and CXCR4-Directed RLT}

RLT was preceded in every patient by a pretherapeutic dosimetry study with $200 \mathrm{MBq}$ of ${ }^{177} \mathrm{Lu}$-pentixather intended to identify critical 
TABLE 1

Patients' Characteristics

\begin{tabular}{|c|c|c|c|c|c|c|c|c|c|c|c|}
\hline $\begin{array}{l}\text { Patient } \\
\text { no. }\end{array}$ & Sex & $\begin{array}{l}\text { Age } \\
(\mathrm{y})\end{array}$ & Disease & $\begin{array}{l}\text { Previous } \\
\text { lines of } \\
\text { therapy }\end{array}$ & $\begin{array}{l}\text { Conditioning } \\
\text { regimen }\end{array}$ & $\begin{array}{l}\text { Additional } \\
\text { radioimmuno- } \\
\text { therapy }\end{array}$ & $\begin{array}{c}\text { RLT to } \\
\text { neutropenia }\end{array}$ & $\begin{array}{c}\text { SCT to } \\
\text { neutrophil } \\
\text { reconstitution }\end{array}$ & $\begin{array}{c}\text { Best } \\
\text { response }\end{array}$ & $\begin{array}{c}\text { Progression- } \\
\text { free } \\
\text { survival }\end{array}$ & $\begin{array}{l}\text { Overall } \\
\text { survival }\end{array}$ \\
\hline 1 & $\mathrm{M}$ & 62 & DLBCL & 2 & $\begin{array}{l}\text { Fludarabine, } \\
\text { busulfan, } \\
\text { thiotepa, } \\
\text { ATG }\end{array}$ & No & 8 & 9 & NA & 29 & 29 \\
\hline 2 & $\mathrm{~F}$ & 46 & DLBCL & 3 & $\begin{array}{c}\text { Fludarabine, } \\
\text { busulfan, } \\
\text { thiotepa }\end{array}$ & No & 15 & 10 & Mixed & 110 & 334 \\
\hline 3 & $\mathrm{~F}$ & 64 & DLBCL & 5 & $\begin{array}{l}\text { Fludarabine, } \\
\text { busulfan, } \\
\text { thiotepa, } \\
\text { ATG }\end{array}$ & No & 7 & NA & NA & 34 & 34 \\
\hline 4 & $M$ & 47 & DLBCL & 3 & $\begin{array}{l}\text { Fludarabine, } \\
\text { busulfan, } \\
\text { thiotepa, } \\
\text { ATG }\end{array}$ & No & 7 & 13 & Mixed & 34 & 62 \\
\hline 5 & $\mathrm{~F}$ & 52 & DLBCL & 3 & $\begin{array}{l}\text { Busulfan, } \\
\text { thiotepa, } \\
\text { ATG }\end{array}$ & $\begin{array}{l}\text { Yes }\left({ }^{188} \text { Re-anti- }\right. \\
\text { CD66) }\end{array}$ & 5 & 13 & PR & 89 & 89 \\
\hline 6 & $M$ & 55 & DLBCL & 6 & $\begin{array}{l}\text { Fludarabine, } \\
\text { treosulfan, } \\
\text { thiotepa, } \\
\text { ATG }\end{array}$ & Yes (90Y-Zevalin) & 20 & 11 & PR & 109 & 115 \\
\hline
\end{tabular}

ATG = antithymocyte globulin; NA = not applicable.

All time intervals are given in days.

organs and the safely administrable activities and to estimate the achievable tumor doses as outlined previously (21).

On the basis of their individual dosimetry, patients were treated by intravenous injection of ${ }^{90}$ Y-labeled pentixather (Table 2). ${ }^{90}$ Y-pentixather was used for treatment to enhance cross irradiation of potential areas of reduced uptake in extramedullary lesions and, more importantly, to shorten the time to SCT. With its 64 -h physical half-life, ${ }^{90} \mathrm{Y}$ activity and thus the residual absorbed dose are reduced to $2.6 \%$ of the total absorbed dose after 2 wk by physical decay only. The effective half-life of ${ }^{90}$ Y-pentixather in the red marrow is even shorter, typically not exceeding $2.5 \mathrm{~d}$. The residual dose to the red marrow is therefore less than 0.75 Gy $14 \mathrm{~d}$ after ${ }^{90}$ Y-pentixather treatment with $30 \mathrm{~Gy}$ of red marrow dose, and SCT can be safely administered.

RLT was performed at a median of $4 \mathrm{~d}$ (range, 2-23 d) after pretherapy dosimetry. To reduce renal toxicity, $2 \mathrm{~L}$ of an amino acid solution containing arginine and lysine $(25 \mathrm{~g} / \mathrm{L}$ each) were coinfused in accord with the joint International Atomic Energy Agency, European Association of Nuclear Medicine, and Society of Nuclear Medicine and Molecular Imaging practical guidance on receptor radionuclide therapy in neuroendocrine tumors (22). Kidney protection by amino acids has been reported to reduce kidney uptake of ${ }^{177} \mathrm{Lu}$-pentixather by about $45 \%$ (20). Vital signs, complete blood count, and blood chemistry were documented during the infusion and within $7 \mathrm{~d}$ after administration.

\section{Concomitant Radioimmunotherapy}

In 2 patients, radioimmunotherapy aiming at CD20 for direct targeting of CD20-positive lymphoma cells or CD66 for indirect targeting of bone marrow infiltration was added to enhance antilymphoma activity. For targeting CD20 on DLBCL cells, a $15 \mathrm{MBq} / \mathrm{kg}$ dose of ${ }^{90}$ Y-ibritumomab tiuxetan (Zevalin; Spectrum Pharmaceuticals) was infused $7 \mathrm{~d}$ after administration of CXCR4-directed RLT, preceded by rituximab at a dose of $250 \mathrm{mg} / \mathrm{m}^{2}$ of body surface area 1 wk before and on the day of radioimmunotherapy.

In the patient receiving additional radioimmunotherapy targeting CD66 because of high-risk lymphoma with initial bone marrow infiltration (and loss of CD20), the murine anti-CD66 monoclonal antibody BW 250/183 (anti-Granulocyte; Scintec Diagnostics) was labeled with ${ }^{188} \mathrm{Re}$ as previously described (23) and delivered from the Department of Nuclear Medicine (radiopharmaceutical division) of Dresden University Hospital. Two days after ${ }^{90}$ Y-pentixather treatment, anti-CD66-targeted radioimmunotherapy with 5.6 GBq (maximum amount available) of ${ }^{188} \mathrm{Re}$-anti-CD66-BW250/183 was performed. Favorable antibody distribution was verified using whole-body planar scintigraphy $44 \mathrm{~h}$ after radioimmunotherapy.

\section{Conditioning Chemotherapy and Stem Cell Transplantation}

All patients except patient 2 received stem cell grafts from unrelated donors. Therefore, in these subjects additional conditioning chemotherapy included busulfan (in $n=5$ patients)/treosulfan ( $n=$ 1), antithymocyte globulin (ATG Fresenius S; Neovii), fludarabine, and thiotepa. In patient 5 , fludarabine was withheld because of chronic kidney failure.

\section{Safety and Response Assessment}

Adverse events were classified as in the Medical Dictionary for Regulatory Activities (24); severity was assessed according to the National Cancer Institute Common Terminology Criteria for Adverse Events (25).

If available (in $n=3$ patients), response was defined according to the Lugano criteria (26) after 6, 11, and $16 \mathrm{wk}$. Progression-free survival was defined as the time from RLT until disease progression, initiation of new antitumor therapy, or death. 
TABLE 2

Overview of Administered Activities and Estimated Organ and Lymphoma Doses

\begin{tabular}{|c|c|c|c|c|c|c|}
\hline \multirow[b]{2}{*}{ Parameter } & \multicolumn{6}{|c|}{ Patient no. } \\
\hline & 1 & 2 & 3 & 4 & 5 & 6 \\
\hline \multicolumn{7}{|l|}{ Pretherapy dosimetry } \\
\hline 177Lu-pentixather (MBq) & 224 & 224 & 200 & 208 & 200 & 211 \\
\hline Kidney max_mL (Gy/GBq $\left.{ }^{177} \mathrm{Lu}\right)$ & 0.8 & 2.9 & 1.3 & 0.8 & * & 1.3 \\
\hline Liver (Gy/GBq $\left.{ }^{177} \mathrm{Lu}\right)$ & 0.2 & 0.6 & 0.7 & 0.4 & 0.6 & 0.9 \\
\hline Spleen (Gy/GBq $\left.{ }^{177} \mathrm{Lu}\right)$ & 0.3 & 0.8 & 0.7 & 1.0 & 1.7 & 0.7 \\
\hline Bone marrow max_mL (Gy/GBq $\left.{ }^{177} \mathrm{Lu}\right)$ & $\dagger$ & $\dagger$ & * & 4.8 & 2.4 & 0.9 \\
\hline Extramedullary lesion max_mL (Gy/GBq $\left.{ }^{177} \mathrm{Lu}\right)$ & 0.9 & 1.9 & 4.5 & & 5.5 & 1.9 \\
\hline \multicolumn{7}{|l|}{ RLT } \\
\hline${ }^{90}$ Y-pentixather (GBq) & 5.3 & 2.8 & 4.3 & 6.5 & 5.8 & 6.2 \\
\hline Added RLT with ${ }^{188} \mathrm{Re}$-anti-CD66 (GBq) & & & & & 5.6 & \\
\hline Added RLT with ${ }^{90} \mathrm{Y}-$ Zevalin (GBq) & & & & & & 1.4 \\
\hline \multicolumn{7}{|l|}{ Posttherapy dosimetry ${ }^{\ddagger}$} \\
\hline Kidney max_mL (no protection) (Gy/GBq ${ }^{90} \mathrm{Y}$ ) & 3.5 & 11.3 & 4.9 & 3.8 & * & 5.7 \\
\hline Liver (Gy/GBq $\left.{ }^{90} \mathrm{Y}\right)$ & 0.9 & 1.8 & 1.6 & 1.3 & 1.6 & 2.0 \\
\hline Spleen (Gy/GBq $\left.{ }^{90} \mathrm{Y}\right)$ & 1.2 & 1.8 & 1.6 & 3.4 & 3.6 & 1.8 \\
\hline Bone marrow (Gy/GBq $\left.{ }^{90} \mathrm{Y}\right)$ & $\dagger$ & $\dagger$ & * & 11.4 & 5.1 & 1.9 \\
\hline Extramedullary lesion max_mL (Gy/GBq $\left.{ }^{90} \mathrm{Y}\right)$ & 3.9 & 5.3 & 10.4 & & 16.7 & 7.1 \\
\hline Kidney max_mL (with protectionף) (Gy) & 11.2 & 19.2 & 12.9 & 14.6 & * & 20.9 \\
\hline Liver (Gy) & 5.0 & 5.1 & 7.1 & 8.4 & 9.5 & 12.2 \\
\hline Spleen (Gy) & 6.6 & 5.2 & 7.0 & 21.8 & 20.7 & 11.1 \\
\hline Bone marrow max_mL (Gy) & $\dagger$ & $\dagger$ & * & 73.8 & 29.4 & 11.7 \\
\hline Extramedullary lesion max_mL (Gy) & 20.9 & 15.0 & 45.2 & & 96.5 & 43.5 \\
\hline \multicolumn{7}{|l|}{$\begin{array}{l}{ }^{*} \text { Not evaluable because of malignant structures. } \\
{ }^{\dagger} \mathrm{No} \text { visible tracer accumulation. } \\
{ }^{+} 90 \mathrm{Y} \text {-pentixather, recalculated from pretherapeuti } \\
{ }^{t} 40 \% \text { rection of absorbed radiation } \text { rose of }\end{array}$} \\
\hline
\end{tabular}

\section{RESULTS}

\section{Patient Characteristics}

Between November 2015 and February 2017, 6 patients with advanced-stage, progressive DLBCL were enrolled. All subjects had been extensively pretreated and had undergone multiple lines of previous treatment (2-6 lines of treatment; median, 3 lines). At the time of presentation, multiple lymphoma manifestations in lymph nodes ( $n=5$ patients), bone $(n=3)$, lungs $(n=3)$, kidneys $(n=3)$, pleura $(n=2)$, spleen $(n=2)$, and muscle $(n=2)$, as well as in adrenal, pancreas, stomach, and thyroid $(n=1$ each), were documented.

\section{Pretherapeutic Dosimetry}

The activities of ${ }^{177} \mathrm{Lu}$-pentixather administered for the pretherapeutic dosimetry study and the measured specific absorbed doses in the kidneys, liver, spleen, bone marrow, and tumor are listed in Table 2. The kidneys were the dose-limiting organ for all patients (apart from expected myeloablation). Lymphoma doses of up to $5.5 \mathrm{~Gy} / \mathrm{GBq}$ of ${ }^{177} \mathrm{Lu}$-pentixather $\left(16.7 \mathrm{~Gy} / \mathrm{GBq}\right.$ of ${ }^{90}$ Y-pentixather) were deduced.

\section{Therapy and Adverse Events}

${ }^{90} \mathrm{Y}$ was administered as the radionuclide of choice in all patients. Because of the shorter half-life of ${ }^{90} \mathrm{Y}$ than of ${ }^{177} \mathrm{Lu}$, the residual dose in the medullary cavities is reliably below $750 \mathrm{mGy}$ after an interval of $14 \mathrm{~d}$ between RLT and subsequent SCT (in contrast to a much longer and more heterogeneous interval with $\left.{ }^{177} \mathrm{Lu}\right)$. The tolerable activity of ${ }^{90}$ Y-pentixather was estimated from the kinetics measured in the pretherapeutic dosimetry targeting at a 23-Gy kidney dose in the 1-mL volume with the highest measured activity concentration (max_mL). The actually infused activities $\left(2.8-6.4 \mathrm{GBq}\right.$ of $\left.{ }^{90} \mathrm{Y}\right)$, the tolerable activities without kidney protection, and the estimated actually absorbed doses in the kidney max_mL (assuming a 40\% kidney dose reduction due to the amino acid solution) are listed in Table 2 for each patient.

During RLT application, no immediate toxic or allergic reactions or changes in vital signs occurred. No acute adverse renal, hepatic, or cardiac events were observed.

As expected, RLT (in combination with additional conditioning chemotherapy) resulted in neutropenia of less than $500 / \mu \mathrm{L}$ after a median of $8 \mathrm{~d}$ (range, 5-20 d) and complete myeloablation after a median of $13 \mathrm{~d}$ (range, $8-23 \mathrm{~d}$ ) in all patients. 
Allogeneic SCT was performed after a median of $17.5 \mathrm{~d}$ (range, 14-25 d).

Successful engraftment with an absolute neutrophil recovery of more than 500 could be observed in 5 of 6 patients after a median of $11 \mathrm{~d}$ (range, 9-13 d). The remaining patient (patient 3) experienced serious infectious complications during aplasia and died from fungal sepsis $34 \mathrm{~d}$ after RLT.

\section{Antilymphoma Activity}

Patient 1 died from central nervous system aspergillosis $29 \mathrm{~d}$ after RLT. Of the remaining 4 of 6 patients available for response assessment, a partial response was recorded for two (patients 5 and 6; Fig. 1) and a mixed response for the remaining two (patients 2 and 4). Both patients with a partial response had undergone CXCR4-directed RLT in combination with radioimmunotherapy.

Noteworthy, patient 2 presented with a complete remission of all CXCR4-positive lymphoma manifestations but the appearance of new receptor-negative lesions (associated with lumbar spine nerve roots), which were exclusively detected by ${ }^{18}$ F-FDG PET/CT (Fig. 2).

During follow-up, another patient (patient 5) died of pneumogenic sepsis (while being in partial remission), and the remaining three (patients 2, 4, and 6) succumbed to lymphoma progression. Median progression-free survival was $62 \mathrm{~d}$ (range, 29-110 d), and median overall survival was $76 \mathrm{~d}$ (range, 29-334 d). In total, 3 patients died of infectious complications (patients 1 and 3 during aplasia, patient 5 after initial successful engraftment 89 d after RLT), and the remainder died of disease progression.

\section{DISCUSSION}

After the initial reports of the feasibility of RLT with ${ }^{177} \mathrm{Lu}-$ and ${ }^{90}$ Y-labeled pentixather in patients with relapsed or refractory multiple myeloma, this is the first description, to our knowledge, of CXCR4-directed radionuclide therapy in relapsed or refractory DLBCL. In parallel with our previous experience with RLT in multiple myeloma and acute leukemia (19), ${ }^{90}$ Y-pentixather as part of the conditioning regimen before allogeneic SCT was well tolerated and could readily be combined with additional treatment, including chemotherapy or radioimmunotherapy targeting CD20

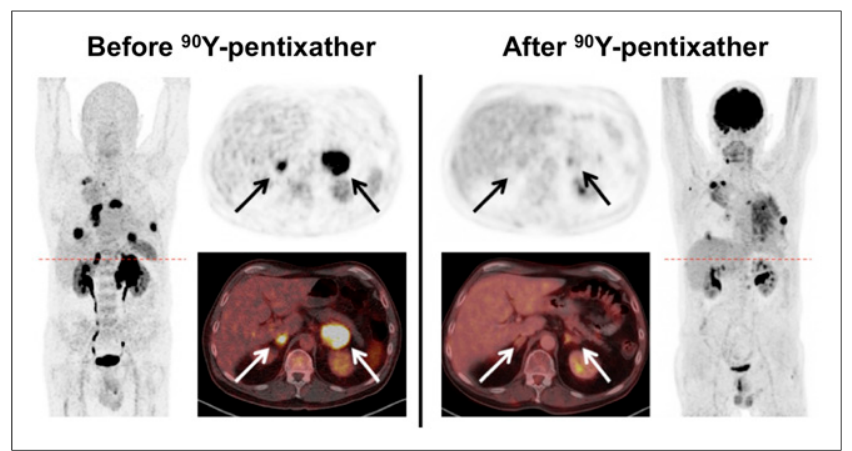

FIGURE 1. Example of partial response after CXCR4-targeted RLT (and additional radioimmunotherapy with ${ }^{90} \mathrm{Y}$-Zevalin) as part of conditioning regimen before allogeneic SCT in DLBCL (patient 6). Shown is display of maximum-intensity projections (outer columns) and transaxial slices (inner columns, with PET at top and PET/CT at bottom) of pretherapeutic CXCR4-directed and posttherapeutic ${ }^{18} \mathrm{~F}-\mathrm{FDG}$ PET/CT. Post-RLT imaging was performed $11 \mathrm{wk}$ after SCT and demonstrated partial response of renal, adrenal (arrows), pulmonary, and most nodal DLBCL manifestations. Diffuse uptake on ${ }^{18} \mathrm{~F}$-FDG PET in left lung was caused by inflammatory changes due to pneumonia.

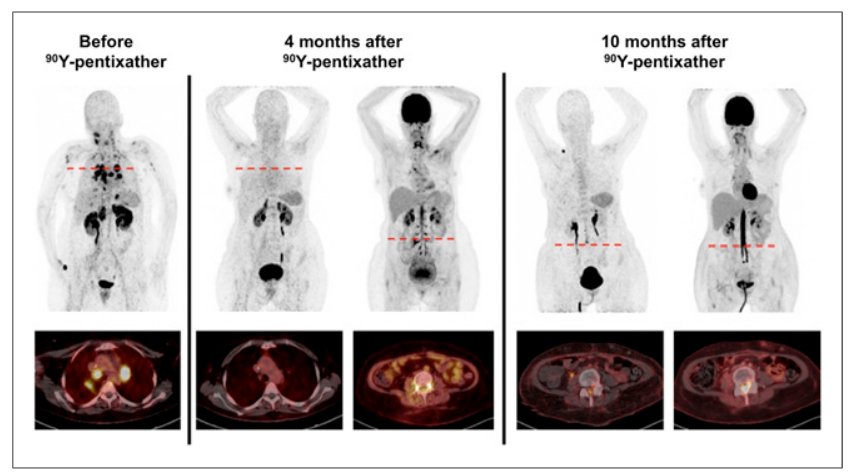

FIGURE 2. Emergence of viable, CXCR4-negative DLBCL after RLT. Shown is display of maximum-intensity projections and transaxial fused PET/CT images of patient 2 before, 4 mo after, and 10 mo after CXCR4directed RLT with ${ }^{90} \mathrm{Y}$-pentixather. Whereas RLT resulted in complete remission of all known lymph node, pulmonary, and adrenal lesions (as compared with pretherapeutic MRI), patient complained about new-onset bladder dysfunction. Additional ${ }^{18} \mathrm{~F}$-FDG PET/CT revealed emergence of new viable, CXCR4-negative lymphoma manifestations affecting spinal cord as well as dorsal root ganglia. Chemotherapy with trofosfamide was started. After initial response, restaging revealed progressive disease with numerous new paraspinal (still CXCR4-negative) lesions.

or CD66. Apart from the expected hematotoxicity resulting in myeloablation, no acute renal, hepatic, or cardiac adverse events were observed. No toxic or allergic reactions occurred. Because of infectious complications associated with aplasia, 2 patients died from fungal sepsis 29 and $34 \mathrm{~d}$ after RLT. Transplantation-related mortality was therefore within the reported ranges for allogeneic SCT and was not further increased by the addition of RLT. Successful donor stem cell engraftment could be recorded 10-13 d after transplantation and was not compromised by preceding radionuclide therapy.

An objective partial or at least mixed response could be observed in all patients available for response assessment. Noteworthy, the 2 patients who underwent additional conditioning radioimmunotherapy were those who achieved partial remission, and one might therefore speculate that patients could benefit from even further intensified treatment before SCT. However, this very preliminary finding needs to be confirmed in larger trials.

In this small cohort, treatment efficiency correlated with estimated absorbed tumor doses. The 2 patients with an objective response were estimated to achieve the highest lymphoma doses of more than 90 Gy (patient 5) and more than 40 Gy (patient 6) (and additional radioimmunotherapy).

However, response duration was rather short-lived in all cases, with a median progression-free survival of only $62 \mathrm{~d}$. These results are quite comparable to the observations of our pilot studies in multiple myeloma, with an initial objective treatment response in more than $80 \%$ of cases but disease relapse in all cases after a median of $54 \mathrm{~d}(21)$. These rather disappointing results might directly reflect patient selection, with all subjects undergoing RLT after exhaustion of all alternative treatments. Given the fact that all patients presented with very advanced, highly refractory lymphoma, more durable responses would have been achieved at earlier disease stages or by adding consolidating or maintaining therapies directly after tumor-debulking CXCR4-directed RLT to better address lymphoma heterogeneity and to eradicate surviving DLBCL cell clones. Tumor heterogeneity or clonal evolution could impressively be demonstrated in one patient who presented 
with complete disappearance of all former, initially CXCR4expressing, lymphoma manifestations but emergence of new, chemokine receptor-negative, lesions. Although we have recently reported on the dynamic regulation of CXCR4 on the tumor cell surface in response to (bridging) chemotherapy (27), the phenomenon of receptor downregulation of a therapy-resistant subclone has not been observed yet. Future basic studies to further investigate and elucidate the underlying pathways are urgently needed. A better understanding of CXCR4 biology might also lead to the identification of synergistic therapy combinations to better address tumor heterogeneity and improve the efficacy and duration of CXCR4-directed RLT. Additionally, introduction of $\alpha$-labeled therapeutic vectors (e.g., ${ }^{213}$ Bismuth- or ${ }^{225}$ Ac-labeled pentixather) could help to overcome the resistance of subclones as recently demonstrated for highly refractory prostate cancer (28-30). Over the short term, new insights will be generated in the COLPRIT trial (Eudra-CT 2015-001817-28), which will focus on the potential role of CXCR4-directed RLT in multiple myeloma and other lymphoproliferative cancers.

This study had several limitations. It was retrospective and comprised a very limited number of patients. Treatment protocols were not identical for all patients. Because of the obvious need to combine RLT with subsequent stem cell support, the antilymphoma efficacy of ${ }^{90}$ Y-pentixather as a single agent could not be reliably assessed.

\section{CONCLUSION}

CXCR4-directed RLT as a conditioning regimen in relapsed or refractory lymphoma before allogeneic SCT is feasible and able to achieve objective responses in even very advanced disease stages. Given the short response duration, further assessment of this novel treatment earlier in the course of disease and in combination with other treatment approaches to enhance efficacy is needed.

\section{DISCLOSURE}

Hans-Jürgen Wester is founder and shareholder of Scintomics. No other potential conflict of interest relevant to this article was reported.

\section{ACKNOWLEDGMENTS}

We thank Gabriele Bohley, Cornelia Schubert, Monika Siemer, Simone Seifert, Michael Schulze-Glück (members of the nuclear medicine team), Inge Grelle, and the whole staff of Ward M63 for their support and assistance.

\section{REFERENCES}

1. Swerdlow SH, Campo E, Pileri SA, et al. The 2016 revision of the World Health Organization classification of lymphoid neoplasms. Blood. 2016;127:2375-2390.

2. Sehn LH, Donaldson J, Chhanabhai M, et al. Introduction of combined CHOP plus rituximab therapy dramatically improved outcome of diffuse large B-cell lymphoma in British Columbia. J Clin Oncol. 2005;23:5027-5033.

3. Kurtova AV, Tamayo AT, Ford RJ, Burger JA. Mantle cell lymphoma cells express high levels of CXCR4, CXCR5, and VLA-4 (CD49d): importance for interactions with the stromal microenvironment and specific targeting. Blood. 2009;113:4604-4613.

4. Moreno MJ, Bosch R, Dieguez-Gonzalez R, et al. CXCR4 expression enhances diffuse large B cell lymphoma dissemination and decreases patient survival. J Pathol. 2015;235:445-455.

5. Wester HJ, Keller U, Schottelius M, et al. Disclosing the CXCR4 expression in lymphoproliferative diseases by targeted molecular imaging. Theranostics. 2015;5: 618-630.

6. Chen Z, Teo AE, McCarty N. ROS-induced CXCR4 signaling regulates mantle cell lymphoma (MCL) cell survival and drug resistance in the bone marrow microenvironment via autophagy. Clin Cancer Res. 2016;22:187-199.
7. Demmer O, Gourni E, Schumacher U, Kessler H, Wester HJ. PET imaging of CXCR4 receptors in cancer by a new optimized ligand. ChemMedChem. 2011;6: 1789-1791.

8. Gourni E, Demmer O, Schottelius M, et al. PET of CXCR4 expression by a ${ }^{68} \mathrm{Ga}-$ labeled highly specific targeted contrast agent. J Nucl Med. 2011;52:1803-1810.

9. Lapa C, Luckerath K, Kleinlein I, et al. ${ }^{68} \mathrm{Ga}$-pentixafor-PET/CT for imaging of chemokine receptor 4 expression in glioblastoma. Theranostics. 2016;6: 428-434.

10. Lapa $\mathrm{C}$, Luckerath $\mathrm{K}$, Rudelius $\mathrm{M}$, et al. $\left[{ }^{68} \mathrm{Ga}\right]$ pentixafor-PET/CT for imaging of chemokine receptor 4 expression in small cell lung cancer: initial experience. Oncotarget. 2016;7:9288-9295.

11. Vag T, Gerngross C, Herhaus $P$, et al. First experience with chemokine receptor CXCR4-targeted PET imaging of patients with solid cancers. J Nucl Med. 2016;57: 741-746.

12. Philipp-Abbrederis $\mathrm{K}$, Herrmann $\mathrm{K}$, Knop S, et al. In vivo molecular imaging of chemokine receptor CXCR4 expression in patients with advanced multiple myeloma. EMBO Mol Med. 2015;7:477-487.

13. Bluemel C, Hahner S, Heinze B, et al. Investigating the chemokine receptor 4 as potential theranostic target in adrenocortical cancer patients. Clin Nucl Med. 2017;42:e29-e34.

14. Lapa C, Schreder M, Schirbel A, et al. $\left[{ }^{68} \mathrm{Ga}\right]$ pentixafor-PET/CT for imaging of chemokine receptor CXCR4 expression in multiple myeloma: comparison to $\left[{ }^{18} \mathrm{~F}\right] \mathrm{FDG}$ and laboratory values. Theranostics. 2017;7:205-212.

15. Werner RA, Weich A, Higuchi T, et al. Imaging of chemokine receptor 4 expression in neuroendocrine tumors: a triple tracer comparative approach. Theranostics. 2017;7:1489-1498.

16. Lapa C, Kircher S, Schirbel A, et al. Targeting CXCR4 with $\left[{ }^{68} \mathrm{Ga}\right]$ pentixafor: a suitable theranostic approach in pleural mesothelioma? Oncotarget. 2017;8:9673296737.

17. Fang HY, Munch NS, Schottelius M, et al. CXCR4 is a potential target for diagnostic PET/CT imaging in Barrett's dysplasia and esophageal adenocarcinoma. Clin Cancer Res. 2018;24:1048-1061.

18. Schottelius M, Osl T, Poschenrieder A, et al. $\left[{ }^{177} \mathrm{Lu}\right]$ pentixather: comprehensive preclinical characterization of a first CXCR4-directed endoradiotherapeutic agent. Theranostics. 2017;7:2350-2362.

19. Habringer S, Lapa C, Herhaus $P$, et al. Dual targeting of acute leukemia and supporting niche by CXCR4-directed theranostics. Theranostics. 2018;8:369383.

20. Herrmann K, Schottelius M, Lapa C, et al. First-in-human experience of CXCR4-directed endoradiotherapy with ${ }^{177} \mathrm{Lu}$ - and ${ }^{90}$ Y-labeled pentixather in advanced-stage multiple myeloma with extensive intra- and extramedullary disease. J Nucl Med. 2016;57:248-251.

21. Lapa C, Herrmann K, Schirbel A, et al. CXCR4-directed endoradiotherapy induces high response rates in extramedullary relapsed multiple myeloma. Theranostics. 2017;7:1589-1597.

22. Bodei L, Mueller-Brand J, Baum RP, et al. The joint IAEA, EANM, and SNMMI practical guidance on peptide receptor radionuclide therapy (PRRNT) in neuroendocrine tumours. Eur J Nucl Med Mol Imaging. 2013;40:800-816.

23. Ringhoffer M, Blumstein N, Neumaier B, et al. ${ }^{188}$ Re or ${ }^{90}$ Y-labelled anti-CD66 antibody as part of a dose-reduced conditioning regimen for patients with acute leukaemia or myelodysplastic syndrome over the age of 55: results of a phase I-II study. Br J Haematol. 2005;130:604-613.

24. Welcome to MedDRA. Medical Dictionary for Regulatory Activities website. https://www.meddra.org/. Accessed September 5, 2018.

25. Common Terminology Criteria for Adverse Events v3.0 (CTCAE). Cancer Therapy Evaluation Program website. http://ctep.cancer.gov/protocoldevelopment/ electronic_applications/docs/ctcaev3.pdf. Published August 9, 2006. Accessed September 5, 2018.

26. Cheson BD, Fisher RI, Barrington SF, et al. Recommendations for initial evaluation, staging, and response assessment of Hodgkin and non-Hodgkin lymphoma: the Lugano classification. J Clin Oncol. 2014;32:3059-3068.

27. Lapa C, Luckerath K, Kircher S, et al. Potential influence of concomitant chemotherapy on CXCR4 expression in receptor directed endoradiotherapy. $\mathrm{Br} \mathrm{J}$ Haematol. January 24, 2018 [Epub ahead of print].

28. Sathekge M, Knoesen O, Meckel M, Modiselle M, Vorster M, Marx S. ${ }^{213} \mathrm{Bi}-$ PSMA-617 targeted alpha-radionuclide therapy in metastatic castration-resistant prostate cancer. Eur J Nucl Med Mol Imaging. 2017;44:1099-1100.

29. Kratochwil C, Bruchertseifer F, Rathke H, et al. Targeted alpha-therapy of metastatic castration-resistant prostate cancer with ${ }^{225}$ Ac-PSMA-617: dosimetry estimate and empiric dose finding. J Nucl Med. 2017;58:1624-1631.

30. Kratochwil C, Bruchertseifer F, Rathke H, et al. Targeted alpha-therapy of metastatic castration-resistant prostate cancer with ${ }^{225}$ Ac-PSMA-617: swimmer-plot analysis suggests efficacy regarding duration of tumor control. J Nucl Med. 2018;59: 795-802. 